\title{
Comportamento humano na criação de cães e a prevalência de parasitos intestinais com potencial zoonótico
}

\author{
Human behavior in dog breeding and the prevalence of \\ intestinal parasites with zoonotic potential
}

Maria Anete Lallo[a], Diva Denelle Spadacci-Morena ${ }^{[b]}$, Selene Dall'Acqua Coutinho[c]

[a] Pós-Graduação em Patologia Ambiental e Experimental, Universidade Paulista (UNIP), São Paulo, SP - Brasil, e-mail: anetelallo@hotmail.com

[b] Departamento de Fisiopatologia, Instituto Butantan, São Paulo, SP - Brasil, e-mail: diva.morena@butantan.gov.br

[c] Pós-Graduação em Patologia Ambiental e Experimental, Universidade Paulista (UNIP), São Paulo, SP - Brasil, e-mail: selene@uol.com.br

\section{Resumo}

O objetivo do presente estudo foi avaliar a natureza das relações estabelecidas em cães de estimação e seus donos em áreas urbanas com pequenos espaços de convivência como a cidade de São Paulo e seu papel na prevalência de parasitos intestinais com potencial zoonótico. Para tal, amostras fecais de cães atendidos em clínicas veterinárias $(n=500)$, residentes em abrigos $(n=250)$ e em canis comerciais $(n=250)$ foram examinadas para detecção de parasitos intestinais por microscopia de luz e por imunofluorescência direta. Para avaliar as relações estabelecidas entre cães e os responsáveis por eles, foi aplicado questionário aos responsáveis pelos cães atendidos nas clínicas veterinárias pesquisadas. Do total de amostras testadas, $45 \%$ foram positivas para parasitos intestinais, sendo que os cães criados coletivamente em abrigos (57,2\%) e canis (48\%) apresentaram as maiores prevalências de parasitos intestinais, especialmente infecções por protozoários. 0 questionário revelou que a maioria dos cães foi adotada a partir de abrigos públicos, tinham acesso livre a casa e aos quartos, dormiam na cama com seus donos e não recebiam tratamentos antiparasitários regulares. Concluiu-se que os parasitos intestinais foram muito prevalentes em cães. A presença de G. duodenalis, Cryptosporidium sp., Toxocara canis e Ancylostoma sp., todos agentes de zoonoses, sugere que os cães podem ser fonte de infecção para os seres humanos ou para o ambiente. As estreitas relações estabelecidas com os seus cães, demonstrada por hábitos de criação e estilo de vida dos proprietários, podem favorecer a transmissão de zoonoses parasitárias, especialmente pelos hábitos de dormir com o cão ou ser lambido pelo mesmo.

Palavras-chave: Cães. Comportamento humano. Parasitos intestinais. Zoonoses. 


\section{Abstract}

The aim of this study was to evaluate the nature of the relationship established between pet dogs and their owners in urban areas with small living spaces as the city of São Paulo and its role in the prevalence of intestinal parasites with zoonotic potential. For this purpose, fecal samples from dogs presented to veterinary clinics $(n=500)$, residing in shelters $(n=250)$ and in commercial kennels $(n=250)$ were examined for detection of intestinal parasites by light microscopy and by direct immunofluorescence. In order to evaluate the relations between dogs and people responsible for them, a questionnaire was applied to the responsible of the dogs presented in veterinary clinics. From the total samples tested, $45 \%$ were positive for intestinal parasites, and collectively created the dogs in shelters (57.2\%) and kennel (48\%) had the highest prevalence of intestinal parasites, particularly protozoan infections. The questionnaire revealed that most of the dogs were adopted from public shelters, had free access to the house and the rooms, sleeping in bed with their owners and did not receive regular parasite treatments. It is concluded that intestinal parasites have high prevalence in dogs. The presence of Giardia duodenalis, Cryptosporidium sp., Toxocara canis and Ancylostoma sp., all zoonotic agents, suggests that dogs may be a source of infection for humans or the environment. The close relationships with their dogs, demonstrated by people creating habits and lifestyle, may favor the transmission of parasitic zoonoses, particularly the habit of sleeping with the dog or being licked by it.

Keywords: Dogs. Intestinal Parasites. Human behavior. Zoonoses.

\section{Introdução}

Os parasitos intestinais estão entre os agentes patogênicos mais comuns encontrados pelos veterinários dedicados ao tratamento dos animais de estimação e constituem uma das principais causas de doenças de alta morbidade em cães (Martínez-Moreno et al., 2007). Vários parasitos que infectam os cães apresentam transmissão entre espécies e se constituem como zoonoses, as quais são altamente prevalentes em seres humanos (Chomel e Sun, 2011). Esses agentes de zoonoses impactam a saúde pública nos países em desenvolvimento e estão associados a condições sanitárias precárias (Overgaauw et al., 2009).

Entre os parasitos intestinais com potencial zoonótico que merecem destaque, são citados os protozoários Giardia sp. e Cryptosporidium sp., e os helmintos Ancylostoma sp. e Toxocara sp.. Giardia duodenalis (sin. G. intestinalis ou $G$. lamblia) e Cryptosporidium são patógenos com grande risco para a saúde pública, uma vez que podem ser transmitidos pela água e pelo contato direto com animais infectados. Os cistos de Giardia e oocistos de Cryptosporidium são eliminados em sua forma infectante, assim o contato direto com pelame desses animais, pode representar uma via importante de transmissão, particularmente para indivíduos imunossuprimidos, os quais são mais suscetíveis (Taylor et al., 2007). Por outro lado, os ovos de helmintos, em especial Toxocara canis e ancilostomídeos, necessitam de condições ambientais apropriadas para se tornarem infectantes no meio ambiente (Kirkpatrick, 1988); logo, o contato direto é menos importante. Entretanto, deve ser ressaltado que animais positivos eliminam ovos e o contato com o ambiente contaminado possibilita a transmissão da infecção zoonótica.

A urbanização da sociedade levou a mudanças na relação entre os seres humanos e os seus animais de estimação. A vida solitária e a restrição de espaço físico aproximaram o homem e seu animal de estimação, os quais têm dividido pequenos ambientes (Macpherson, 2005).

Nos Estados Unidos, $61 \%$ das residências possuem animais de estimação, sendo que 39\% têm cães, $31 \%$ possuem mais de um cão e $50 \%$ das famílias que possuem cães também têm crianças. Os dados revelam que $56 \%$ dos proprietários de cães dormem próximos de seus animais e 50\% dormem com eles na cama (Chomel e Sun, 2011). Recentemente, um estudo mostrou que $50 \%$ dos proprietários recebem lambidas de seus cães na face, $60 \%$ dos cães frequentam o quarto, $45 \%$ ficam na cama do proprietário e 18\% dormem com seus proprietários na cama (Overgaauw et al., 2009). 
Na cidade de São Paulo (Brasil), uma pesquisa revelou a proporção de um cão para cada 4,34 pessoas e a presença 1,6 cães por residência. Nas residências que possuem cães, existem cerca de 4 habitantes e $81,5 \%$ dos proprietários possuem cães para companhia. Esse mesmo estudo revelou que a maioria dos cães foram adotados (73,9\%) e um número pequeno de animais foram comprados $(26,1 \%)$ (Canatto et al., 2012).

Muitos estudos realizados mostram a prevalência de parasitos intestinais em cães em todo o mundo, porém pouco se sabe sobre a aproximação dos animais de estimação e seus responsáveis, o que é fundamental para evidenciar a possibilidade de transmissão de zoonoses. Assim, os objetivos desse estudo foram: analisar o comportamento humano na criação de cães de estimação em áreas urbanas com pequenos espaços, como a região metropolitana de São Paulo, as quais poderiam indicar erros de conduta que propiciam a transmissão de zoonoses; e avaliar a prevalência de parasitos intestinais em cães.

\section{Material e métodos}

Entre janeiro de 2012 e dezembro de 2013, foram coletadas amostras fecais $(\mathrm{n}=1.000)$ de cães, domiciliados $(n=500)$, de abrigos $(n=250)$ e de canis comerciais $(n=250)$ para a pesquisa de parasitos intestinais. Os cães domiciliados assim como as clínicas e hospitais veterinários que participaram da pesquisa foram randomicamente selecionados a partir das clínicas que existem na área metropolitana de São Paulo, durante o período do estudo, sendo excluídos os animais com queixas gastrintestinais, imunossuprimidos ou com doenças debilitantes. Os animais de canis e abrigos também foram randomicamente selecionados a partir de informações de sociedades protetoras dos animais (para os abrigos) e do kennel club (para os canis comerciais). Este estudo foi aprovado pelo Comitê de Ética e Pesquisa Animal da Universidade (número do processo 176/2011).

A população estudada consistiu de 460 cães (46\%) sem raça definida, 540 (54\%) de raças puras, sendo 550 (55\%) fêmeas e 450 (45\%) machos. Dezenove raças diferentes estavam presentes e as mais comuns foram Poodle ( $\mathrm{n}=98)$, Dachshund ( $\mathrm{n}$
$=40)$, Cocker spaniel $(\mathrm{n}=25)$, Pinscher $(\mathrm{n}=20) \mathrm{e}$ Rottweiler ( $\mathrm{n}=18)$. A idade média foi 3 anos e 5 meses, com variação de 3 meses até 13 anos (25\% quartil: 1 ano, e $75 \%$ quartil: 6 anos).

As amostras fecais foram coletadas pelos proprietários dos animais domiciliados, após evacuação espontânea. Nos canis e abrigos, os espécimes fecais foram coletados pelos autores, diretamente do reto dos animais. Em seguida, as amostras de fezes foram mantidas em duplicata entre $4-8{ }^{\circ} \mathrm{C}$ por $24-48 \mathrm{~h}$ ou a $-20{ }^{\circ} \mathrm{C}$ até o processamento.

Os espécimes fecais foram macroscopicamente examinados para a detecção de proglotes e fragmentos e/ou parasitos inteiros. Em seguida, foram processadas pelas técnicas de flutuação espontânea (2g de fezes) em solução saturada de cloreto de sódio (s.g. 1.2), centrífugo-flutuação em sacarose saturada (s.g. 1.2) e centrífugosedimentação, método de Ritchie (Allen e Ridley, 1970) e observadas pela microscopia de luz para avaliar a presença de ovos e (oo)cistos de parasitos (Taylor et al., 2007). Todos os ovos de helmintos e formas infectantes de protozoários foram identificados, baseando-se em características morfológicas (Taylor et al., 2007), usando microscopia de luz com amplificações de 100x e 400x.

Foi utilizada a técnica de imunofluorescência direta para a detecção de antígenos de Giardia e Cryptosporidium (IFA; Merifluor Cryptosporidium/ Giardia kit; Meridian Diagnostics Inc.) (Geurden et al., 2008). Para a determinação de oocistos de Cryptosporidium foi utilizada ainda a coloração de Ziehl Neelsen modificada aplicada sobre um esfregaço fecal realizado com o sedimento obtido pela técnica de Ritchie (Henriksen e Pohlenz, 1980). Os cistos de Giardia foram identificados pela técnica de flutuação.

Para entender as relações de interação estabelecidas entre os responsáveis e seus animais e assim avaliar melhor o risco de transmissão de zoonoses foi aplicado questionário contendo 10 questões referentes à forma de criação e interação homem/animal (Quadro 1).

As prevalências e o intervalo de confiança (CI 95\%) foram determinados para cada grupo estudado e para todas as formas parasitárias encontradas comparados pelo teste de T de Student. 
A partir dos resultados dos questionários, foram obtidas as prevalências, intervalo de confiança (CI 95\%) e o risco relativo para as categorias avaliadas. A regressão logística multivariada foi utilizada para quantificar a associação entre parasitos com cada variável (Hosmer e Lemeshow, 1989). Todas as análises estatísticas foram feitas com software SPSS 15.0 para Windows (SPSS, Chicago, Illinois, USA).

Quadro 1 - Questionário aplicado para entender as relações de interação estabelecidas entre os responsáveis e os seus cães, São Paulo, Brasil

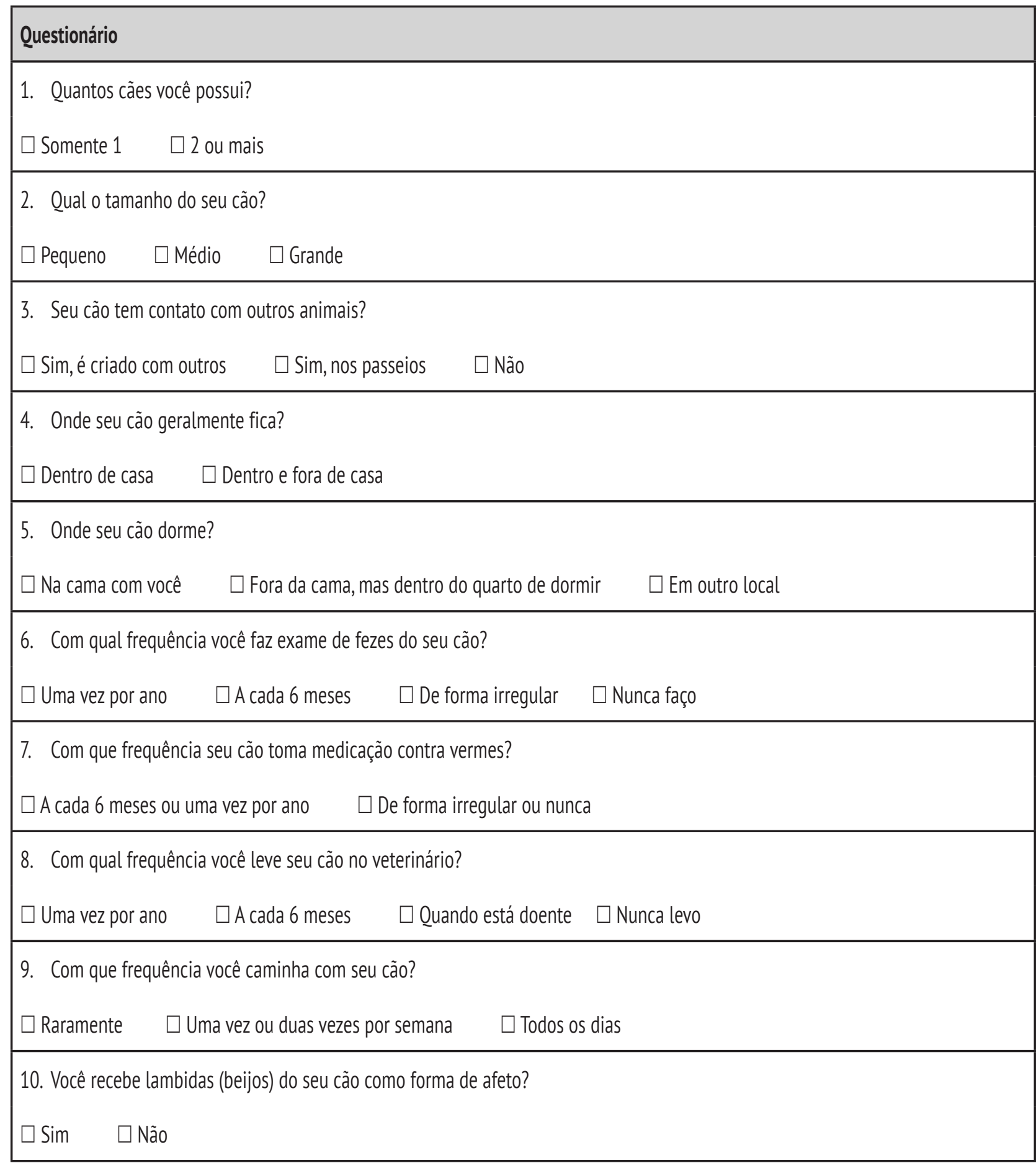




\section{Resultados}

0 questionário evidenciou alguns pontos importantes a respeito do risco de transmissão de zoonoses parasitárias (Tabela 1). A maioria dos proprietários (70\%, 95\% CI: 58-84,6) adquiriu seu cão de estimação pela adoção de abrigos ou diretamente da rua. A análise dos questionários sugere que cães criados com outros cães possuem maior risco de apresentar parasitos intestinais $(R R=2,0 ; 95 \%$ CI: 1,3-3,1) do que cães criados sozinhos. Esse estudo também sugere que cães que possuem acesso à rua também têm maior risco de apresentar parasitos intestinais $(\mathrm{RR}=1,5 ; 95 \% \mathrm{CI}$ : $0,85-2,6)$ do que animais criados somente dentro de casa (indoor). Setenta e um porcento dos animais tinham acesso aos quartos, 57\% (95\% CI: 47,4$63,5)$ dormiam com seus responsáveis em suas camas e 58\% (95\% CI: 46-67) dos responsáveis adoravam beijar ou receber lambidas na face de seus cães. A ausência ou a administração irregular de drogas antiparasitárias pode apresentar maior risco relativo de infecções por parasitos intestinais
( $R R=2,1 ; 95 \%$ CI: 1,1-2,9). Uma questão muito importante é que 93\% (95\% CI: 85-98) dos cães estudados era submetido a exames fecais de forma irregular ou simplesmente não eram submetidos.

A análise estatística indicou que o risco de infecção por parasitos intestinais foi maior em cães jovens ( $R R=4,80 ; 95 \%$ CI: 3,1-6,2) e que viviam coletivamente ( $\mathrm{RR}=1,5 ; 95 \% \mathrm{CI}$ : $0,83-2,9$ ). 0 gênero e a raça dos cães estudados não tiveram efeito significante na prevalência de parasitos intestinais (Tabela 1).

A prevalência total de parasitos intestinais, considerando as três populações estudadas, foi $45 \%$ e entre os animais positivos $62,4 \%$ estavam infectados por apenas uma espécie de parasito, enquanto 35,6\% tinham duas espécies diferentes de parasitos e somente alguns animais $(5,3 \%)$ tinham mais que duas espécies diferentes de parasitos. A prevalência de parasitos foi significantemente maior em cães criados coletivamente em abrigos (57,2\%, 95\% CI: 48-67) ou canis (48\%, 95\% CI: 4358) do que em cães domiciliados $(37 \%, 95 \% \mathrm{CI}$ : 27-39).

Tabela 1 - Fatores de risco associados à presença de parasitas intestinais de acordo com a idade, gênero e condições de vida em cães analisados em São Paulo, Brasil

\begin{tabular}{lccc}
\multicolumn{1}{c}{ Variável } & Risco Relativo (RR) & $\begin{array}{c}\text { Intervalo de } \\
\text { confiança de } \\
95 \% \text { para o RR }\end{array}$ & Valor de P \\
\hline Idade: jovem/adulto & 4,8 & $3,1-6,2$ & $0,003^{*}$ \\
Gênero: machos/fêmeas & 1,0 & $0,47-1,53$ & NS \\
Raça: raça pura/SRD & 1,3 & $0,78-1,62$ & NS \\
$\begin{array}{l}\text { Condições de vida: } \\
\text { a) Abrigos ou canis/domiciliados }\end{array}$ & 1,5 & & $0,01^{*}$ \\
b) 2 ou mais cães/ um cão na residência & 2,0 & $0,83-2,9$ & $0,02^{*}$ \\
c) Fora de casa/dentro de casa & 1,5 & $1,3-3,1$ & $0,01^{*}$ \\
$\begin{array}{l}\text { d) Ausência ou uso irregular } \\
\text { antiparasitários/ uso regular }\end{array}$ & 2,1 & $0,85-2,6$ & $0,01^{*}$ \\
\hline
\end{tabular}

Legenda: SRD, sem raça definida; NS, não significante.

Nota: *Estatisticamente significante $P<0,05$. 
As infecções por protozoários (39\%, 95\% CI: 31,4-59,7) foi significantemente maior do que a infecção por helmintos (18,7\%, 95\% CI: 12,3-25,6). Giardia duodenalis e Isospora (I. canis e I. ohiensislike) foram os protozoários mais prevalentes (Tabela 2). Os cistos de G. duodenalis foram detectados pela microscopia de luz em 200 dos 1.000 cães $(\mathrm{P}=20 \%)$ e os antígenos específicos foram identificados por IFA em 345 de 1.000 amostras (34,5\%). A prevalência pela IFA foi significativamente maior em abrigos ( $\mathrm{P}=38 \%, 95 \%$ CI: $32,6-42,5)$ e canis $(40 \%, 95 \%$ CI: $35.6-43.2)$ do que nos cães domiciliados $(\mathrm{P}=20 \%, 95 \% \mathrm{CI}$ :
18,8-24,2). Identificou-se maior prevalência de ovos de ascarídios e ancilostomídeos, enquanto foram baixas as prevalências de T. vulpis e $D$. caninum (Tabela 2). Os cães criados em canis tiveram alta prevalência de protozoários e prevalência baixa de helmintos.

\section{Discussão}

Muitos parasitos intestinais de cães são causadores de zoonoses consideradas importantes para a saúde pública, por exemplo, Toxocara canis,

Tabela 2 - Prevalência (\%) de parasitos intestinais em cães de diferentes populações, São Paulo, Brasil

\begin{tabular}{|c|c|c|c|}
\hline \multirow[b]{2}{*}{ Parasitos } & \multicolumn{3}{|c|}{ População de cães } \\
\hline & $\begin{array}{c}\text { Domiciliados ( } n=500) \\
\% \text { (IC) }\end{array}$ & $\begin{array}{c}\text { Abrigos ( } n=250) \\
\text { P\% (IC\%) }\end{array}$ & $\begin{array}{c}\text { Canis comerciais }(n=250) \\
\text { P\% (IC\%) }\end{array}$ \\
\hline Ancylostoma spp. & $16(12,2-24,1)$ & $26(20,1-34,3)^{a}$ & $10(6,5-14,8)$ \\
\hline Toxocara canis & $11(8,8-15,7)$ & $12,8(8,2-16,2)$ & $8(4,3-10,6)$ \\
\hline Trichuris vulpis & $3(1,7-4,6)$ & $3,6(1,7-5,3)$ & $2,8(1,9-3,9)$ \\
\hline Dipylidium caninum & $1,6(0,2-2,1)$ & $2(0,8-4,1)$ & $0,8(0,1-1,3)$ \\
\hline Giardia spp. & $20(18,8-24,2)^{b}$ & $38(32,6-42,5)$ & $40(35,6-43,2)$ \\
\hline Isospora spp. & $13,3(10,2-15,2)$ & $18(14,1-21,2)$ & $20(16,2-24,2)$ \\
\hline Hammondia heydorni & $3,6(2,2-4,9)$ & $6,4(4,1-9,6)$ & $4,8(3,1-6,9)$ \\
\hline Sarcocystis spp. & $3(1,9-4,8)$ & $4,8(2,4-6,3)$ & $4(2,7-6,2)$ \\
\hline Cryptosporidium spp. & $6(4,7-8,6)$ & $2,8(1,5-4,2)$ & $5,2(3,7-6,6)$ \\
\hline Total de parasitos & $37(27-39)^{c}$ & $57,2(48-67)$ & $48(43-58)$ \\
\hline
\end{tabular}

Legenda: a Diferença significante na prevalência de Ancylostoma spp. entre cães de canis comerciais e domiciliados $(P<0,002)$; ${ }^{b}$ Diferença significante na prevalência de $G$. duodenalis entre cães de abrigos e canis $(P<0,001)$; ' Diferença significante na prevalência total de parasitos de cães domiciliados comparados aos de abrigo e canis $(P<0.01)$.

Nota: IC, 95\% intervalo de confiança. 
Giardia duodenalis e Cryptosporidium spp. Todos esses parasitos intestinais possuem rota de transmissão oral-fecal e o maior componente para a disseminação é a presença de ovos ou (oo)cistos no meio ambiente. A análise dos questionários respondidos pelos proprietários mostrou que a adoção de cães errantes ou provenientes de abrigos é a maior fonte de aquisição dos animais, um fato que reforça a necessidade de cuidados especiais com esses animais que constituem fontes de infecção para os seus proprietários. Além disso, nesse trabalho evidenciou-se as altas prevalências de parasitos em animais e abrigos e canis, locais onde os animais são adquiridos. Assim, adoção de medidas preventivas pelos veterinários pode auxiliar na diminuição da transmissão de zoonoses, por exemplo, indicação de exames parasitológicos de fezes, administração de drogas antiparasitárias, banhos e quarentena.

Entretanto, essa pesquisa identificou que a administração de drogas antiparasitárias é irregular ou simplesmente não acontece. Adicionalmente, a realização de exames fecais foi incipiente, o que pode ajudar a explicar esse cenário de alta prevalência de parasitos. 0 custo financeiro dessas atitudes pode ser muito alto para a sociedade brasileira. 0 conhecimento do ciclo de vida dos parasitos, sua epidemiologia e profilaxia fazem com que veterinários tenham papel fundamental como promotores de saúde, responsáveis pela educação dos proprietários de animais de estimação.

A prevalência de parasitos foi alta em cães que vivem em coletividade e isto foi reforçado pelo risco maior de parasitose intestinal em cães que conviviam com outros cães em domicílios, avaliado pelo questionário. Adicionalmente, cães criados fora de casa ou com acesso à rua também tiveram maior prevalência de parasitos. Em áreas urbanas, onde os animais de companhia têm que dividir com os outros animais espaços limitados, como parques públicos, praias, praças de cães e outros espaços de recreação, a possibilidade de transmissão e disseminação de parasitoses é alta (Chomel e Sun, 2011). No Brasil, os espaços públicos também são compartilhados com cães errantes, o que aumenta a contaminação ambiental.

No presente estudo, hábitos de criação com muita proximidade foram identificados. 0 risco de transmissão de agentes de zoonoses pelo contato próximo entre proprietários e seus pets dividindo a cama, por beijos ou lambidas é real e tem sido relatado. Beijar os animais de estimação também pode transmitir zoonoses. Um estudo realizado no Japão com 24 proprietários de pets (11 gatos e 13 cães) não encontrou Pasteurella spp. na cavidade oral de 19 proprietários que não tinham beijado seus gatos, porém isolou $P$. stomatis da cavidade oral de 1 dos 2 proprietários que haviam beijado seu gato e em 2 de 3 proprietários de cães que também tinham beijado seus cães (Arashima et al., 1992). Enquanto ovos e oocistos de alguns parasitos necessitam de um tempo no meio ambiente para se tornarem infectantes, para outros como Cryptosporidium e Giardia, os oocistos e cistos, respectivamente, são eliminados na forma infectante e contaminam pelos, sendo facilmente transmitidos pelo contato com as mãos ou membranas mucosas de seus proprietários. Para reduzir tais riscos, a educação dos proprietários pelo veterinário é fundamental.

A prevalência geral de parasitos intestinais nos cães estudados (45\%) foi similar às encontradas em estudos anteriores realizados em diferentes países, por exemplo, foi 39,2\% em Portugal (Ferreira et al., 2011), 35,5\% na Venezuela (Ramirez-Barrios et al., 2004), 52,4\% na Argentina (Fontanarrossa et al., 2006) e 53\% na Hungria (Fok et al., 2011). No presente estudo, infecções com uma única espécie de parasito foi mais prevalente, assim como observado por outros autores (Kirkpatrick, 1988; Ramirez-Barrios et al., 2004).

A prevalência de parasitos intestinais é variável e depende de um grande número de fatores incluindo idade, condições de vida, método de diagnóstico empregado, região estudada e estado de saúde do animal (Tangtrongsup e Scorza, 2010). Os parasitos intestinais foram principalmente encontrados em cães de abrigos e canis, em contraste com os cães domiciliados. Estes achados são muito consistentes com os encontrados por outras pesquisas em que os índices de infecção intestinal foram mais altos nos animais mantidos em abrigos (Palmer etal., 2008; Scaramozzino et al., 2009). De maneira especial em abrigos, um grande número de cães costuma ser mantido em espaços limitados, o que contribui para a propagação de parasitos, aumentando o risco de infecção (Bajer et al., 2011). A alta prevalência em canis comerciais, 
como foi aqui observada, indica que provavelmente os mesmos fatores predisponentes estão presentes, tais como a superpopulação, falhas no manejo e controle sanitário.

Nesse estudo, a prevalência de protozoários foi maior que de helmintos, em todas as populações estudadas. Comumente, os animais de companhia residentes em abrigos estão sob a supervisão de veterinários, sendo vacinados e tratados com antihelmínticos para prevenir a disseminação de doenças infecciosas e parasitárias, o mesmo acontece em cães criados em canis ou domiciliados. Entretanto, parasitos protozoários não são incluídos nesses tratamentos, fato que pode explicar a maior prevalência de protozoários (Scaramozzino et al., 2009).

Nesse estudo, Giardia foi o parasito mais prevalente, particularmente em abrigos e canis, mostrando que os fatores acima expostos estão presentes; porém, a veiculação hídrica de Giardia também deve ser considerada como fator fundamental na epidemiologia dessa parasitose.

A prevalência de Cryptosporidium spp. em cães foi similar a outros estudos realizados no Canadá e pelo mundo (Palmer et al., 2008; Uehlinger et al., 2013). Em contraste, a prevalência de Cryptosporidium spp. não variou significantemente de acordo com a população estudada (Palmer et al., 2008). Embora a prevalência de Cryptosporidium tenha sido baixa, existe grande preocupação em relação a este protozoário por se tratar de um agente oportunista, responsável por diarreia grave em indivíduos imunossuprimidos (Xiao e Feng, 2008). Muitos casos de infecção humana têm sido associados ao genótipo bovino de Cryptosporidium parvum e, mais recentemente, $C$. canis foi identificado em casos humanos, sugerindo forte ligação entre as infecções humanas e animal (Xiao e Feng, 2008).

Os helmintos mais prevalentes foram Ancylostoma sp. e Toxocara canis. Outros autores encontraram T. canis e ancilostomídeos (Uncinaria sp. e Ancylostoma sp.) como os helmintos mais comuns em cães (Kirkpatrick, 1988). Em particular, esses helmintos têm maior relevância para a saúde pública devido ao contato próximo entre as pessoas e seus animais de estimação e porque são causadores de zoonoses reconhecidas, larva migrans cutânea e visceral (Kirkpatrick, 1988).
Nesse estudo, os animais mais jovens tiveram maior prevalência que os adultos (considerados com mais de 1 ano), o que coincide com outros estudos (Bridger e Whitney, 2009; Little et al., 2009). Muitos fatores justificam a maior prevalência nos jovens. Em relação aos helmintos, as transmissões transmamária e transplacentária para os filhotes são os grandes responsáveis pelas altas prevalências em T. canis e Ancylostoma sp. em filhotes (Zajac e Conboy, 2007). A aquisição de imunidade da mucosa intestinal é fator limitante para o desenvolvimento de protozoários e pode explicar a diminuição da prevalência em adultos.

\section{Conclusão}

Os parasitos intestinais se mostraram frequentes nos cães avaliados neste estudo. Os animais jovens, criados coletivamente, que frequentam as ruas e não são tratados regularmente com antiparasitários apresentaram aqui maior prevalência de parasitos intestinais. A presença de G. duodenalis e Cryptosporidium spp. sugere que cães podem ser fonte de infecção de giardíase e criptosporidiose para humanos. Toxocara canis e Ancylostoma spp. foram prevalentes e poderiam ser fontes de contaminação ambiental. A relação próxima dos cães e seus proprietários aqui avaliados foi muito alta, o que poderia favorecer a transmissão de zoonoses, sobretudo, dormir e beijar os animais.

\section{Agradecimentos}

Nós agradecemos os proprietários de clínicas, abrigos e canis que gentilmente contribuíram com a realização desse estudo, e aos proprietários que responderam ao questionário.

\section{Referências}

Allen AV, Ridley DS. Further observations on the formolether concentration technique for faecal parasites. Journal of Clinical Pathology. 1970; 23(6):545-546. 
Arashima Y, Kumasaka K, Okuyama K, Kawabata M, Tsuchiya T, Kawano $\mathrm{K}$ et al. Clinicobacteriological study of Pasteurella multocida as a zoonosis (1). Condition of dog and cat carriers of Pasteurella, and the influence for human carrier rate by kiss with the pets. Kansenshogaku Zasshi. 1992; 66(2):221-224.

Bajer A, Bednarska M, Rodo A. Risk factors and control of intestinal parasites infections in sled dogs in Poland. Veterinary Parasitology. 2011; 175(3-4):343-350. doi:10.1016/j.vetpar.2010.10.029.

Bridger KE, Whitney H. Gastrointestinal parasites in dogs from the Island of St. Pierre off the south coast of Newfoundland. Veterinary Parasitology. 2009; 162(12):167-170. doi:10.1016/j.vetpar.2009.02.016.

Canatto BD, Silva EA, Bernardi F, Mendes MCNC, Paranhos NT, Dias RA. Caracterização demográfica das populações de cães e gatos supervisionados do município de São Paulo. Arquivo Brasileiro de Medicina Veterinária e Zootecnia. 2012; 64(6):15151523. doi:10.1590/S0102-09352012000600017.

Chomel BB, Sun B. Zoonoses in the bedroom. Emerging Infectious Diseases. 2011; 17(2):167-172.

Ferreira FS, Pereira-Baltasar P, Parreira R, Padre L, Vilhena M, Távora Tavira L et al. Intestinal parasites in dogs and cats from the district of Évora, Portugal. Veterinary Parasitology. 2011; 179(1-3):242-245. doi:10.1016/j.vetpar.2011.02.003.

Fok E, Szatmári V, Busák K, Rozgonyi F. Prevalence of intestinal parasites in dogs in some urban and rural areas of Hungary. The Veterinary Quarterly. 2011; 23(2):96-98.

Fontanarrossa MF, Vezzani D, Basabe J, Eiras DF. An epidemiological study of the gastrointestinal parasites of dogs from Southern Greater Buenos Aires (Argentina): age, gender, breed, mixed infections, and seasonal and spatial patterns. Veterinary Parasitology. 2006; 136(3-4):283-295. doi:10.1016/j. vetpar.2005.11.012.

Geurden T, Berkvens D, Casaert S, Vercruysse J, Claerebout E. A Bayesian evaluation of three diagnostic assays for the detection of Giardia duodenalis in symptomatic and asymptomatic dogs. Veterinary Parasitology. 2008; 157(1-2):14-20. doi:10.1016/j. vetpar.2008.07.002.
Henriksen SA, Pohlenz JFL. Staining of Cryptosporidia by a Ziehl-Neelsen technique. Acta Veterinaria Scandinavica. 1980; 22(3-4):594-596.

Hosmer DW, Lemeshow S. Applied logistic regression. New York: Wiley; 1989.

Kirkpatrick CE. Epizootiology of endoparasitic infections in pet dogs and cats presented to a veterinary teaching hospital. Veterinary Parasitology. 1988; 30(2):113-124.

Little SE, Johnson EM, Lewis D, Jaklitsch RP, Payton ME, Blagburn BL et al. Prevalence of intestinal parasites in pet dogs in the United States. Veterinary Parasitology. 2009; 166(1-2):144-152. doi:10.1016/j. vetpar.2009.07.044.

Macpherson CNL. Human behaviour and the epidemiology of parasitic zoonoses. International Journal of Parasitology. 2005; 35(11-12):1319-1331. doi:10.1016/j.ijpara.2005.06.004.

Martínez-Moreno FJ, Hernández S, López-Cobos E, Becerra C, Acosta I, Martínez-Moreno A. Estimation of canine intestinal parasites in Córdoba (Spain) and their risk to public health. Veterinary Parasitology. 2007; 143(1):7-13. doi:10.1016/j.vetpar.2006.08.004.

Overgaauw PA, van Zutphen L, Hoek D, Yaya FO, Roelfsema J, Pinelli E et al. Zoonotic parasites in fecal samples and fur from dogs and cats in the Netherlands. Veterinary Parasitology. 2009; 163(1-2):115-122. doi:10.1016/j.vetpar.2009.03.044.

Palmer CS, Traub RJ, Robertson ID, Devlin G, Rees R, Thompson RCA. Determining the zoonotic significance of Giardia and Cryptosporidium in Australian dogs and cats. Veterinary Parasitology. 2008; 154(1-2):142-147. doi:10.1016/j.vetpar.2008.02.031.

Ramírez-Barrios RA, Barboza-Mena G, Muñoz J, AnguloCubillán F, Hernández E, González F et al. Prevalence of intestinal parasites in dogs under veterinary care in Maracaibo, Venezuela. Veterinary Parasitology. 2004; 121(1-2):11-20. doi:10.1016/j.vetpar.2004.02.024.

Scaramozzino P, Di Cave D, Berrilli F, D’Orazi C, Spaziani A, Mazzanti S et al. A study of the prevalence and genotypes of Giardia duodenalis infecting kennelled dogs. The Veterinary Journal. 2009; 182(2):231-234. doi:10.1016/j.tvjl.2008.07.003. 
Tangtrongsup S, Scorza V. Update on the diagnosis and management of Giardia spp. infections in dogs and cats. Topics in Companion Animal Medicine. 2010; 25(3):155-162. doi:10.1053/j.tcam.2010.07.003.

Taylor MA, Coop RL, Wall RL. Veterinary parasitology. 3. ed. Oxford: Blackwell Publishing; 2007.

Uehlinger FD, Greenwood SJ, McClure JT, Conboy G, O'Handle YR, Barkema HW. Zoonotic potential of Giardia duodenalis and Cryptosporidium spp. and prevalence of intestinal parasites in young dogs from different populations on Prince Edward Island, Canada. Veterinary Parasitology. 2013; 196(3-4):509514. doi:10.1016/j.vetpar.2013.03.020.
Xiao L, Feng Y. Zoonotic cryptosporidiosis. FEMS Immunology and Medical Microbiology. 2008; 52(3):309-323. doi:10.1111/j.1574-695X.2008.00377.x.

Zajac AM, Conboy GA. Veterinary clinical parasitology. 7. ed. Ames, IA: Blackwell Publishing Professional; 2007.

Recebido em: 19/01/2016 Received in: 01/19/2016

Aprovado em: 22/07/2016

Approved in: 07/22/2016 